Pacific Journal of Mathematics

ROUND QUADRATIC FORMS UNDER ALGEBRAIC 


\title{
ROUND QUADRATIC FORMS UNDER ALGEBRAIC EXTENSIONS
}

\section{BURKHARD AlPERS}

\begin{abstract}
Pfister forms over fields are those anisotropic forms that remain round under any field extension. Here, round means that for any represented element $x \neq 0$ the isometry $x \varphi \cong \varphi$ holds where $\varphi$ is the form under consideration. We investigate whether a similar characterization can be given for the round forms themselves. We obtain several "going-up" and "going-down" theorems. Some counterexamples are given which show that a general theorem holds neither in the going-up nor in the going-down situation.
\end{abstract}

Introduction. Pfister forms over fields can be characterized as those anisotropic forms that remain round under any field extension (cf. [16, p. 153]). In this paper we investigate whether a similar characterization can be given for round forms over fields. Since the structure of round forms is not known in general, one cannot expect general results. According to a theorem of Marshall [14] (see also Becker/Köpping [3]), every round form has a decomposition $\varphi=l \times \psi+\rho$ where $\psi$ is a Pfister form and $\rho$ is torsion. This implies that any round form remains round over the pythagorean closure of the underlying field. In this paper we shall prove the following characterization theorem for certain types of forms or fields: A form $\varphi$ over $F$ is round iff it is round over every proper quadratic extension $K=F(\sqrt{w})$ where $w$ is represented by $\varphi$ over $F$. As to the going-down part of this equivalence the usual techniques (norm principles) allow one to prove many results. The going-up part, however, requires detailed information on the structure of round forms which is available only for certain classes of fields, for example the linked fields. Counter-examples show that in general neither implication of the equivalence is true. For extensions of odd dimension we have the well-known theorem of Springer which yields immediately that a form over $F$ which is round over $K \geq F$ $([K: F] \in 2 \mathbb{N}+3)$ is also round over $F$. In the other direction, nothing is known.

We use the standard terminology as is found in [16]. The fields occurring in this paper are commutative and of characteristic $\neq 2$. $K$ usually denotes a field, $W(K)$ the corresponding Witt ring, and 
$W_{t}(K)$ or simply $W_{t}$ the torsion part of $W(K)$. For (quadratic) forms $\varphi$ and $\psi$ over $K, \varphi \cong \psi(\varphi=\psi)$ will express isometry (equality in the Witt ring). For the anisotropic part of $\varphi$ we write $\varphi_{a}$. The set of non-zero elements of $K$ represented by $\varphi$ is denoted by $D(\varphi)$ and we shall write $S D(\varphi)$ for the semiring generated by $D(\varphi)$. The set of square classes of elements in $D(\varphi)$ is denoted by $\widetilde{D}(\varphi)$. If no misinterpretation can occur we write $x$ for an element $x \in \dot{F}$ and for the corresponding square class $x F^{* 2}$ as well. If $D(\varphi)=K^{*}:=K \backslash\{0\}$, then $\varphi$ is said to be universal. In the case $1 \in D(\varphi)$ the pure subform of $\varphi$ is denoted by $\varphi^{\prime}$, that is $\varphi \cong\langle 1\rangle \perp \varphi^{\prime}$. The form $\varphi$ is called round if $x \cdot \varphi \cong \varphi$ holds for all $x \in D(\varphi)$, that is, $D(\varphi)=G(\varphi)$ where $G(\varphi):=\left\{x \in K^{*} \mid x \varphi \cong \varphi\right\}$. According to this definition, an isotropic form is round if its anisotropic part is 0 or round and universal.

1. Going-up. There are several examples of fields where every round form remains round over any finite field extension. This is true for algebraic number fields, finite fields, and $p$-adic fields since these classes are closed with respect to finite extensions and there are characterizations of round forms which are invariant under field extensions (cf. [2]). But in general there are no such characterizations.

To begin with, we consider the situation $\operatorname{dim} \varphi \in 2 \mathbb{N}+1$. Trivially, the form $\langle 1\rangle$ remains round over any field extension. Now, let $\varphi$ be round and anisotropic over $F$, and $\operatorname{dim} \varphi \in 2 \mathbb{N}+3$. According to [14], $F$ must be formally real pythagorean, and $\varphi \cong\langle 1, \ldots, 1\rangle$. So, there is no proper quadratic extension $F(\sqrt{w})$ where $w \in D_{F}(\varphi)$.

We now want to prove going-up theorems for round forms with a certain structure, in particular for round forms over linked fields. A field $K$ is called linked if the classes of quaternion algebras over $K$ form a subgroup in the Brauer group of $K$ (cf. [9]). Equivalently, any two 2-fold Pfister forms are linked, that is, for forms $\langle\langle a, b\rangle\rangle$ and $\langle\langle c, d\rangle\rangle$ we may assume $a=c$. In general, Pfister forms $\gamma_{1} \cong$ $\left\langle\left\langle a_{1}, \ldots, a_{m}\right\rangle\right\rangle$ and $\gamma_{2} \cong\left\langle\left\langle b_{1}, \ldots, b_{n}\right\rangle\right\rangle \quad(m \leq n)$ are called linked if there are $c_{1}, \ldots, c_{m-1} \in K^{*}$ such that $\gamma_{1} \cong\left\langle\left\langle c_{1}, \ldots, c_{m-1}, d\right\rangle\right\rangle$ and $\gamma_{2} \cong\left\langle\left\langle c_{1}, \ldots, c_{m-1}, d_{m}, \ldots, d_{n}\right\rangle\right\rangle$ for some $d, d_{m}, \ldots, d_{n}$ in $K$. Round forms over linked fields and over fields whose schemes can be constructed using schemes of linked fields and of fields with $u$-invariant $\leq 4$ have been characterized in [1], [2] (for the special terminology concerning schemes see [11], [12]). For this class (denoted by $\mathscr{L}$ ) we have:

THEOREM 1.1 ([1, 2.10, 3.6]). (i) If $\varphi$ is a round and anisotropic form of dimension $2^{v} l,(2, l)=1$, then there exists a decomposition 
$\varphi=l \times \psi+\sum_{i=1}^{m} \rho_{i}$ with a v-fold Pfister form $\psi \notin W_{t} \backslash\{0\}$ and $i$-fold Pfister forms $\rho_{i} \in W_{t}(i=1, \ldots, m), 2^{m} \leq \operatorname{dim} \varphi$, such that for each $i=1, \ldots, m$

$$
\begin{aligned}
& D(\varphi) \subset D(\psi) \cap D\left(\rho_{i}\right) \text { and } \\
& \left(l>1 \Rightarrow D(\varphi)=D(\psi)=D(l \times \psi) \text { and } 2 \rho_{i}=0\right) .
\end{aligned}
$$

(ii) Moreover, if $K$ is linked, then we may assume that there exist an $r \in \mathbb{N}$ and $a_{1}, \ldots, a_{r+v} \in K^{*}$ such that $2^{r+v} \leq \operatorname{dim} \varphi, 2^{r} \times \psi \cong$ $\left\langle\left\langle a_{1}, \ldots, a_{r+v}\right\rangle\right\rangle$ and $\left\langle\left\langle a_{1}, \ldots, a_{i-1}\right\rangle\right\rangle \mid \rho_{i}$ ("simultaneous linkage").

The decomposition of a round form given in (i) is called a Pfisterdecomposition, and any form with such a decomposition is called Pfister-decomposable. For linked fields we can get more information on the sets $D\left(\rho_{i}\right)$. In this case we have $u(K)=\max \left\{\operatorname{dim} \varphi_{a} \mid \varphi \in\right.$ $\left.W_{t}\right\} \leq 8$ (cf. [9]), so we have to examine round forms of the type $\varphi=l \times \psi+\rho_{1}+\rho_{2}+\rho_{3}$. Since $u(K) \leq 8$, the form $\rho_{3}$ is universal. For the other torsion forms we have:

LEMMA 1.2. If $K$ is linked, and if $\varphi$ is a round and anisotropic form over $K$, then there exists a Pfister-decomposition $\varphi=l \times \psi+\sum_{i=1}^{3} \rho_{i}$ with the following additional properties:

(1) If $\varphi \notin W_{t}$, then $D\left(\rho_{i}\right)=K^{*}$ for $i=1,2,3$.

(2) If $\varphi=\rho_{1}+\cdots+\rho_{s} \in W_{t}(s \leq 3)$ with $\rho_{s} \neq 0$, then $D\left(\rho_{1}\right)=$ $D\left(\rho_{s-1}\right)=K^{*}$.

Proof. (i) Assume that the form $\gamma \cong\langle 1, w,-r,-w r\rangle$ is torsion and $D(\gamma)$ contains the semiring generated by $w, r$, and all squares $\neq$ 0 (hence, this semiring does not contain 0 ). Pick an arbitrary $c \in K^{*}$. By the linkage property, we have $D(\langle w,-c,-w c\rangle) \cap D(\langle c, r, c r\rangle) \neq$ $\varnothing$. Therefore, there are $\alpha_{1}, \ldots, \alpha_{t} \in K$ (not all zero) such that $w \alpha_{1}^{2}-c\left(\alpha_{2}^{2}+w \alpha_{3}^{2}\right)=c\left(\alpha_{4}^{2}+r \alpha_{5}^{2}\right)+r \alpha_{6}^{2} \neq 0$. Hence, $w \alpha_{1}^{2}-r \alpha_{6}^{2}=$ $c\left(\alpha_{2}^{2}+\alpha_{4}^{2}+w \alpha_{3}^{2}+r \alpha_{5}^{2}\right)$. Since the RHS is not 0 and $D(\gamma)$ is a group, we have $c \in D(\gamma)$. It follows that $D(\gamma)=K^{*}$.

(ii) Assume that $D(\langle d, a, b, a b\rangle) \subset D(\langle 1,-d\rangle)$ for some $a, b, d$ $\in K^{*}$ (in particular, we have $-1 \in D(\langle 1,-d\rangle)$ ). Pick an arbitrary $c \in K^{*}$. By linkage, we have $D(\langle a d,-c d,-a c\rangle) \cap D(\langle a, c b, c a b\rangle) \neq$ $\varnothing$. Now one can proceed as in (i) to get $D(\langle 1,-d\rangle)=K^{*}$.

(iii) Assume that the form $\langle 1,-d\rangle$ represents any non-zero element of the semiring generated by $d$ and all squares. Applying (ii) with $a=b=1$, we get $D(\langle 1,-d\rangle)=K^{*}$. 
(iv) Assume first $\operatorname{dim} \varphi \geq 6$. Then, $D(\varphi) \cup\{0\}$ is a semiring by $[1,2.9]$. Hence, $\rho_{1}$ is universal by (iii) (if 4 does not divide $\operatorname{dim} \varphi$ we may assume $\rho_{1}=0$; otherwise $\rho_{1}=\langle 1,-d\rangle$ where $d$ is the determinant of $\varphi)$. By linkage, we may assume $\rho_{2} \cong\langle 1, w,-r,-w r\rangle$ where $w, r$ are sums of squares and hence $w, r \in D(\varphi)$. Then (i) gives $D\left(\rho_{2}\right)=K^{*}$. In the remaining case we have $\operatorname{dim} \varphi=4$ but then (ii) yields the desired result.

As a simple application of the linkage property we get:

LEMMA 1.3. If $\psi \neq 0$ and $\rho$ are different linked Pfister forms, $\operatorname{dim} \psi \geq \operatorname{dim} \rho$, and if $\rho$ is universal, then $D(\psi)=D\left((\psi+\rho)_{a}\right)$.

Induction leads to:

Corollary 1.4. Let $\psi$ be a Pfister form and $\rho_{i}$ be $i$-fold universal Pfister forms $(i=1, \ldots, m)$ where $\operatorname{dim} \psi \geq \operatorname{dim} \rho_{m}, \psi \neq \rho_{m}$. If these Pfister forms are simultaneously linked, then $D(\psi)=$ $D\left(\left(\psi+\sum_{i=1}^{m} \rho_{i}\right)_{a}\right)$.

The following lemma is a generalization of [10, 2.18].

LEMMA 1.5. Let $\varphi$ be a round form over $F$ with $D(\varphi)=S D(\varphi) \backslash\{0\}$, and let $K=F(\sqrt{w})$ be a quadratic extension where $w \in D_{F}(\varphi)$. If $\varphi$ is round over $K$, then $D_{K}(\varphi)=S D_{K}(\varphi) \backslash\{0\}$.

Proof. We want to apply [10, 2.17] to $S:=S D_{K}(\varphi) \backslash\{0\}$. Obviously, $S \leq K^{*}$. Now, let $x \in S \cap F$. Since $D_{K}(\varphi)$ is a group we may assume that there are $x_{1}, \ldots, x_{s} \in D_{K}(\varphi)$ with $x=\sum_{i=1}^{s} x_{i}$. Each of the $x_{i}$ is of the type $\sum_{j=1}^{n} c_{j}\left(a_{i j}+b_{i j} \sqrt{w}\right)^{2}$ where $\varphi \cong\left\langle c_{1}, \ldots, c_{n}\right\rangle$. So $x \in D_{F}(\varphi)$ and hence $S \cap F=D_{F}(\varphi)$. Now, let $x \in S$. Again we may assume that there are $x_{1}, \ldots, x_{s} \in D_{K}(\varphi)$ such that $\sum_{i=1}^{s} x_{i}=x$. Hence, $x \in D_{K}\left(\langle 1,1\rangle^{s} \otimes \varphi\right)$. By [16, 2.10.4], $\langle 1,1\rangle^{s} \otimes \varphi$ is round over $K$ and over $F$ as well. By $[\mathbf{1 0}, 2.11]$ we have $N(x) \in G_{F}\left(\langle 1,1\rangle^{s} \otimes \varphi\right)=$ $D_{F}\left(\langle 1,1\rangle^{s} \otimes \varphi\right)=D_{F}(\varphi)$. Thus, $N(S) \subset D_{F}(\varphi) \subset D_{F}(\varphi) \cdot D_{F}(\varphi)$. Applying [10, 2.17], we get $S=D_{K}(\varphi) \cdot(S \cap F)=D_{K}(\varphi) \cdot D_{F}(\varphi)=$ $D_{K}(\varphi)$ since $\varphi$ is round over $K$.

Corollary 1.6. Let $\psi$ be a Pfister form over $F, l \in 2 \mathbb{N}+3$, and let $K=F(\sqrt{w})$ where $w \in D_{F}(l \times \psi)$. If $l \times \psi$ is round over $F$, then $l \times \psi$ is round over $K$. 
Proof. If $l \times \psi$ is round and isotropic over $F$, then it is easy to see that $\psi$ is $F$-universal and the claim follows from [10, 2.15]. Otherwise apply (1.5) and [14, 1.7].

In the sequel we want to improve (1.6) in order to allow the torsion part of the round form to be non-zero. We need the notions of rigid and basic elements (cf. [5], [6]). An element $x \in F^{*}$ is called rigid if $D(\langle 1, x\rangle)=F^{* 2} \cup x F^{* 2}$. A rigid element $x$ is called one-sided rigid (resp. birigid) if $-x$ is non-rigid (resp. rigid). If $x$ is not birigid or if $x \in \pm F^{* 2}$, then $x$ is called basic. As usual, the set of basic elements is denoted by $A(F)$ (the definition in [5] is slightly different from that given in [6] but this does not affect the results we need).

Let $\varphi$ be an anisotropic round form over $F$ with Pfister-decomposition $\varphi=l \times \psi+\sum_{i=1}^{k} \rho_{i}$. It is easy to check that we can write $\varphi$ as a product $\varphi=\left(l \times \alpha+\sum_{i=1}^{k} \delta_{i}\right) \otimes\left\langle\left\langle t_{1}, \ldots, t_{m}\right\rangle\right\rangle$ with birigid elements $t_{j}$ (different modulo $A(F)$ ) where $\psi=\alpha \otimes\left\langle\left\langle t_{1}, \ldots, t_{m}\right\rangle\right\rangle$, $\rho_{i}=\delta_{i} \otimes\left\langle\left\langle t_{1}, \ldots, t_{m}\right\rangle\right\rangle, \gamma:=\left(l \times \alpha+\sum_{i=1}^{k} \delta_{i}\right)_{a}$ is a round form with Pfister-decomposition $l \times \alpha+\sum \delta_{i}, D(\gamma) \subset A(F)$ and, moreover, $D(\alpha) \subset A(F)$ (resp. $D\left(\delta_{i}\right) \subset A(F)$ ) if $\alpha$ (resp. $\delta_{i}$ ) is not 0 . Note that $D_{F}\left(\rho_{i}\right) \supset A(F)$ and $\rho_{i} \neq 0$ imply $D\left(\delta_{i}\right)=A(F)$.

THEOREM 1.7. Let $\varphi$ be a round and anisotropic form over $F$ with Pfister-decomposition $l \times \psi+\sum_{i=1}^{k} \rho_{i}$ and let $K=F(\sqrt{c})$ where $c \in D_{F}(\varphi)$. If $D_{F}(\varphi)=S D_{F}(\varphi) \backslash\{0\}$ and $D_{F}\left(\rho_{i}\right) \supset A(F)$ for $i=$ $1, \ldots, k$, then $\varphi$ is round over $K$.

Proof. First assume that $\varphi$ is universal. Then $\psi=0$ and the $\rho_{i}$ are universal since $\psi \notin W_{t} \backslash\{0\}$ and $D(\varphi) \subset D\left(\rho_{i}\right)$. Now, by $[10,2.15], \rho_{i}$ is universal over $K$ for $i=1, \ldots, k$. Thus, $\varphi$ is a universal round form over $K$ (possibly $=0$ in the Witt ring). Therefore we may assume that $D_{F}(\varphi)=S D_{F}(\varphi)$ (and hence $D_{F}(\psi)=$ $\left.S D_{F}(\psi)\right)$, that is, $D_{F}(\varphi) \cup\{0\}$ is a proper preordering. Let $\varphi=$ $\left(l \times \alpha+\sum_{i=1}^{k} \delta_{i}\right) \otimes\left\langle\left\langle t_{1}, \ldots, t_{m}\right\rangle\right\rangle$ and $\gamma=\left(l \times \alpha+\sum_{i=1}^{m} \delta_{i}\right)_{a}$ according to the above remarks. Then, by [6, Prop. 5], $D_{F}(\gamma)=S D_{F}(\gamma)$ and $D_{F}(\alpha)=S D_{F}(\alpha)$. To begin with, let $K=F(\sqrt{w})$ where $w \in D_{F}(\gamma)$. By $[16,2.10 .4]$, it suffices to prove that $\gamma$ is round over $K$. By (1.6), we have $D_{K}(\alpha)=S D_{K}(\alpha) \neq K$; hence $D_{K}(\gamma) \subset D_{K}(\alpha) \subset$ $A(K)$. Let $\delta \in\left\{\delta_{1}, \ldots, \delta_{k}\right\}$, and assume $\delta_{K}$ is not isotropic. We want to show that $A(K)=D_{K}(\delta)$. By [6, Prop. 2] and [5, 2.2], we have $D_{K}(\delta) \subset A(K)$. We want to apply [10, 2.17] with $S:=$ $A(K)$. By [6, Th. 1], $A(K)$ is a group. Since $w \in D(\gamma) \subset A(F)$, 
[7, 5.6] implies $A(K) \cap F^{*}=A(F)$. From [6, Prop. 1] it follows that $N(A(K)) \subset D_{F}(\langle 1,-w\rangle) \subset A(F)=D_{F}(\delta)$. Hence, [10, 2.17] yields $A(K)=D_{K}(\delta) \cdot\left(A(K) \cap F^{*}\right)=D_{K}(\delta) \cdot D_{F}(\delta)=D_{K}(\delta)$. Therefore, $D_{K}(\gamma) \subset D_{K}(\alpha), D_{K}\left(\delta_{i}\right)$, which means that $\gamma$ is round over $K$. Now, assume that $m \geq 1$ and that $w \in D_{F}(\alpha) \cdot D_{F}\left(\left\langle\left\langle t_{1}, \ldots, t_{m}\right\rangle\right\rangle\right) \backslash A(F)$, say (wlog) $w=a t_{1}$ where $a \in D_{F}(\alpha)$. Then, $a \equiv t_{1} \bmod K^{* 2}$ and $t_{1} \in A(K)$ by [5, 2.3]. This gives

$$
\begin{aligned}
\delta_{i} & \otimes\left\langle\left\langle t_{1}, \ldots, t_{m}\right\rangle\right\rangle \cong_{K} \delta_{i} \otimes\left\langle\left\langle a, t_{2}, \ldots, t_{m}\right\rangle\right\rangle \\
& \cong_{K}\left(\delta_{i} \perp a \delta_{i}\right) \otimes\left\langle\left\langle t_{2}, \ldots, t_{m}\right\rangle\right\rangle \\
& \cong_{K}\left(2 \delta_{i}\right) \otimes\left\langle\left\langle t_{2}, \ldots, t_{m}\right\rangle\right\rangle=0
\end{aligned}
$$

for $i=1, \ldots, k$. Therefore, $\varphi_{K} \cong l \times \psi_{K}$. Since $D_{F}(\psi)=S D_{F}(\psi)$ the proof is complete by (1.6).

COROllary 1.8. If $\varphi$ is a Pfister-decomposable form over $F$ with $D_{F}(\varphi) \cup\{0\}$ containing an ordering of $F$, then $\varphi$ remains round over $F(\sqrt{w})$ whenever $w \in D_{F}(\varphi)$. In particular, if $\varphi$ is a Pfisterdecomposable universal form over $F$, then $\varphi$ is round over any quadratic extension of $F$.

Remarks 1.9. (1) We cannot omit the condition on $D_{F}(\varphi)$ in the preceding theorem: Using quadratic form scheme theory (cf. [11] and the explanations in $\S 2$ ), one can show that there is a non-formally real field $F$ such that there exist universal anisotropic forms $\langle\langle a, b\rangle\rangle$ and $\langle 1,-d\rangle$ with $-1 \not \equiv-d \not \equiv-a \in D_{F}\left(\langle\langle a, b\rangle\rangle^{\prime}\right)$ and $a \not \equiv 1$ (in quadratic form scheme notation: Consider $F$ where $S(F) \cong S\left(\mathbb{Q}_{2}\right) \sqcap S\left(F_{3}\right)$ ). Then, $\varphi \cong\langle d, a, b, a b\rangle$ is round over $F$ and hence over $F((t))$. But for $K:=F((t))(\sqrt{a})$ we have $\varphi_{K} \cong\langle 1,-d, 1,-1\rangle$ and $t \notin$ $D_{K}(\langle 1,-d\rangle)$. Hence, $\varphi$ is not round over $K$.

(2) Naturally, the question arises whether the "process can be continued", i.e., whether the form $\varphi$ remains round over another field extension $L=K(\sqrt{c})$ where $c \in D_{K}(\varphi)$, and so on. But it is clear from the proof of (1.7) that $\varphi_{K}$ retains the properties used in the proof. Therefore, $\varphi$ remains round over any $2^{n}$-extension $K_{n}$ where $K_{n} \geq \cdots \geq K_{1} \geq K_{0}=F$ and $K_{i}=K_{i-1}(\sqrt{w})$ with $w \in D_{K_{t-1}}(\varphi)$ (we call such an extension a $\varphi-2^{n}$-extension). This means in particular that $\varphi$ remains round over any $2^{n}$-extension within the pythagorean closure of $F$ and hence over any extension within the pythagorean closure. 
THEOREM 1.10. If $\varphi$ is a round form over a linked field $F$, then $\varphi$ remains round over $K=F(\sqrt{w})$ whenever $w \in D_{F}(\varphi)$.

Proof. Let $w \in D_{F}(\varphi)$ and $K=F(\sqrt{w})$. According to (1.2), there exists a Pfister-decomposition $\varphi=l \times \psi+\sum_{i=1}^{m} \rho_{i}$ with either $\psi \neq 0$ and $D\left(\rho_{i}\right)=F^{*}$ for $i=1,2,3=m$ or $\psi=0$ and $\rho_{m} \neq 0$ and $D\left(\rho_{i}\right)=F^{*}$ for $i<m \leq 3$. In the first case, the $\rho_{i}$ are universal over $K$, by $[10,2.15]$. Then, in case of $l>1$, we have $D_{F}(\varphi)=D_{F}(\psi)=$ $S D_{F}(\psi)$ and the preceding theorem can be applied. In case of $l=1$, $\varphi$ is round over $K$ by (1.4). If $\psi=0$, we can again apply (1.4) and $[10,2.15]$, thus completing the proof.

REMARK 1.11. Again, the proof of (1.10) depends only on the existence of a special Pfister-decomposition of the round form. It is clear from the proof that the necessary properties are preserved under the quadratic extensions under consideration. Therefore, the process can be continued. Furthermore, if $l=1$, then $\varphi$ remains round over $K$ whenever $K$ lies in the quadratic closure of $F$.

2. Going-down. We shall use different hypotheses depending on the fields under consideration:

(1) $\varphi$ is round over every quadratic extension $F(\sqrt{w})$ where $w \in$ $D_{F}(\varphi)$;

(2) $\varphi$ is round over every quadratic extension $F(\sqrt{w})$ where $w \in$ $\left\langle D_{F}(\varphi)\right\rangle$ (the group generated by $D_{F}(\varphi)$ );

(3) $\varphi$ is round over every quadratic extension $F(\sqrt{w})$ where $w \in$ $-D_{F}(\varphi)$.

Our proofs depend heavily on the possibility of representing form values as norms with respect to a quadratic extension $K \geq F$ and on extending orderings. For this reason, we must look at more extensions than originally intended (cf. (2)) or at different extensions (cf. (3)). (1) and (2) coincide if the form under consideration is a group form (note that from the "going-up" point of view it would be no restriction to require that $\varphi_{F}$ is a group form!). Condition (3), however, is different and yields in general no "going-down" converse for the "going-up" theorems proved in $\S 2$. Moreover, its "going-up" converse is wrong in general. Nevertheless, this condition yields the best "going-down" result. To see this, we need the following result that applies the norm principles (stated in [10]) to the "going-down" situation.

Proposition 2.1. Let $\varphi$ be a form over $F$ and $1, x \in D_{F}(\varphi)$. If there is a $z \in F^{*} \backslash F^{* 2}$ such that $x \in D(\langle 1,-z\rangle)$, and if $\varphi$ is round over $F(\sqrt{z})$, then $x \in G_{F}(\varphi)$. 
Proof. Since $x \in D(\langle 1,-z\rangle)$, there exists a $u \in K:=F(\sqrt{z})$ such that $N(u)=x$. Since $1 \in D_{F}(\varphi),[10,2.13]$ yields $u \in F \cdot D_{K}(\varphi)$. Hence, there is a $\lambda \in F^{*}$ with $\lambda u \in D_{K}(\varphi)$. By [10, 2.11], $\lambda^{2} x=$ $N(\lambda u) \in G_{F}(\varphi)$.

Corollary 2.2 (cf. $[10,3.14])$. Let $\varphi$ be a form over $F$ where $1 \in$ $D_{F}(\varphi) \not \supset-1$. Assume that $\varphi$ fulfills condition (3). Then $\varphi$ is round over $F$.

ReMARK 2.3. The condition $1 \in D_{F}(\varphi)$ cannot be omitted. Consider, for example, the form $\varphi \cong\langle\varepsilon, \pi\rangle$ over a $p$-adic field where $\varepsilon$ is a unit $\not \equiv 1, \pi$ is a prime, and $1 \equiv-1$. The condition $-1 \notin$ $D_{F}(\varphi)$ can be dropped in all cases except one. Assume that $\varphi$ is a form over $F$ with $1,-1 \in D_{F}(\varphi)$ which fulfills condition (3). By (2.1), we get $D_{F}(\varphi) \backslash-F^{* 2} \subset G_{F}(\varphi)$. Now, assume there exists an $a \in \widetilde{D}_{F}(\varphi) \backslash\{ \pm 1\}$. Then, from $a \in G_{F}(\varphi)$ it follows that $-1 \cdot a \in \widetilde{D}_{F}(\varphi) \backslash\{ \pm 1\}$ and hence $-a \in G_{F}(\varphi)$. Since $G_{F}(\varphi)$ is a group we have $-1 \in G_{F}(\varphi)$. Thus, if $\varphi$ is not round over $F$, we have $\widetilde{D}_{F}(\varphi)=\{ \pm 1\}$. If $F$ is formally real, then this means that $F$ must be euclidean, and the form $m \times\langle 1\rangle \perp n \times\langle-1\rangle \quad\left(m, n \in \mathbb{N}^{*}, m \neq n\right)$ is really a counter-example in this case. Let $F$ be non-formally real and let $\varphi$ be a counterexample. We may assume $\operatorname{dim} \varphi>2$. Then, $D_{F}(\varphi)=F^{*}$, and hence $\left|F^{*} / F^{* 2}\right|=2$. Thus, we additionally may assume $\operatorname{dim} \varphi \in 2 \mathbb{N}+3$. But by the Diller/Dress-theorem (which is also true in the non-formally real pythagorean case, cf. [10] (3.9)), $K \neq K^{2}$ for any quadratic extension $K \geq F$. It follows that $\varphi$ is not round over $F(\sqrt{-1})$, but $1 \in D_{F}(\varphi)$, a contradiction to condition (3). We summarize: If $F$ is not euclidean or the form under consideration is anisotropic, then we can omit the condition $-1 \notin D_{F}(\varphi)$ in (2.2). So, for universal forms we get:

COROLlARY 2.4. If $\varphi$ is a universal form over $F$ and anisotropic in the case $F$ is euclidean, and if condition (1) $(\Leftrightarrow(2) \Leftrightarrow(3))$ holds, then $\varphi$ is round over $F$.

Proposition (2.1) yields an easy proof of a part of a theorem in [10] which concerns the going-down step on condition (1):

THEOREM 2.5 (cf. $[\mathbf{1 0}, 3.6])$. Let $\varphi \cong\left\langle a_{1}, \ldots, a_{n}\right\rangle$ be a form over $F$. If $a_{1}, \ldots, a_{n} \in G_{F}(\varphi)$, and if $\varphi$ is round over every extension $F(\sqrt{a})$ where $a \in D_{F}(\varphi)$, then $\varphi$ is round over $F$. 
Proof. We prove by induction on $l$ that $x \in D_{F}\left(\left\langle a_{1}, \ldots, a_{l}\right\rangle\right)$ implies $x \in G_{F}(\varphi)$. The case $l=1$ is trivial. So, assume $x=y+a_{l+1}$ where $y \in D_{F}\left(\left\langle a_{1}, \ldots, a_{l}\right\rangle\right) \subset G_{F}(\varphi)$. We may assume $x \not \equiv a_{l+1}$. Since $x \in D_{F}\left(\left\langle y, a_{l+1}\right\rangle\right)$, we have $x y \in D(\langle 1, x y\rangle) \cap D\left(\left\langle 1, y a_{l+1}\right\rangle\right) \subset$ $D\left(\left\langle 1,-x a_{l+1}\right\rangle\right)$, by [8, Lemma]. Since $y, a_{l+1} \in G_{F}(\varphi)$, we have $x y, x a_{l+1} \in D_{F}(\varphi)$. Now, (2.1) yields $x y \in G_{F}(\varphi)$ and hence $x \in$ $G_{F}(\varphi)$.

Corollary 2.6. Let $\psi$ be a Pfister form over $F$ and let $\varphi \cong l \times \psi$, $l \in 2 \mathbb{N}+1$. If $\varphi$ is round over every extension $F(\sqrt{a})$ where $a \in$ $D_{F}(\varphi)$, then $\varphi$ is round over $F$.

We now want to show that for certain classes of forms and special classes of fields the hypotheses of Theorem 2.5 can be weakened. For a form $\varphi$ over $F$ there exists a decomposition $\varphi \cong \varphi_{1} \perp \varphi_{2}$ where $\varphi_{1}$ is 0 or has a diagonalization in which the entries are squares or non-rigid elements and $\varphi_{2}$ is 0 or represents only rigid elements $\not \equiv$ 1 . We call any such decomposition where $\operatorname{dim} \varphi_{2}$ is minimal an $\mathrm{nr}$ decomposition of $\varphi$. If there is an nr-decomposition of $\varphi$ with $\varphi_{2}=$ 0 , then we say that $\varphi$ is nr-representable.

THEOREM 2.7. Let $\varphi$ be a form over $F$ with $1 \in D_{F}(\varphi)$ which is round over $F(\sqrt{a})$ whenever $a \in D_{F}(\varphi)$. Then, any non-rigid element of $D_{F}(\varphi)$ lies in $G_{F}(\varphi)$. In particular, if $\varphi_{1} \perp\left\langle b_{1}, \ldots, b_{l}\right\rangle$ is an nr-decomposition of $\varphi$ with $b_{1}, \ldots, b_{l} \in G_{F}(\varphi)$, then $\varphi$ is round over $F$.

Proof. Let $z$ be a non-rigid element in $D_{F}(\varphi) \backslash F^{2}$. Then, $\varphi$ has a subform $\langle 1, c\rangle$ such that $z \in D_{F}(\langle 1, c\rangle)$, and hence $c$ is non-rigid. Pick an arbitrary $x \in \widetilde{D}_{F}(\langle 1, c\rangle) \backslash\{1, c\}$. Now, $x \in D_{F}(\langle 1, x\rangle) \cap$ $D_{F}(\langle 1, c\rangle) \subset D_{F}(\langle 1,-x c\rangle)$, by [8, Lemma], and $x c \in D_{F}(\langle 1, c\rangle) \subset$ $D_{F}(\varphi)$. Thus, by $(2.1), x \in G_{F}(\varphi)$. Since $x c \in \widetilde{D}_{F}(\langle 1, c\rangle) \backslash\{1, c\}$, we have also $x c \in G_{F}(\varphi)$ and hence $c \in G_{F}(\varphi)$. Therefore, $z \in$ $D(\langle 1, c\rangle) \subset G_{F}(\varphi)$.

Corollary 2.8. Let $\varphi$ be a form over $F$ with $1 \in D_{F}(\varphi)$. If $\varphi$ is nr-representable and round over every extension $F(\sqrt{a})$ where $a \in D_{F}(\varphi)$, then $\varphi$ is also round over $F$.

Assume that the radical of $F$ is not $F^{* 2}$ (cf. [4]). It is well known that any element of the radical is represented by every 1-fold Pfister 
form. Thus, using (2.1), we get the desired going-down theorem without any restrictions on rigidity. So, we may restrict ourselves in the sequel to the situation where the underlying field $F$ has a trivial radical (hence the notions of [5] and [6] coincide). We shall now have a closer look at the nonformally real case. We call a form $\varphi$ basic if it has a diagonalization with basic entries. It is well known that in this case the anisotropic part of $\varphi$ represents only basic elements (if any). Furthermore, using the results of [6], we easily get the following fact: Let $\varphi \cong \varphi_{1} \perp \varphi_{2} t_{2} \perp \cdots \perp \varphi_{n} t_{n}$ be a (not necessarily anisotropic) form where the $\varphi_{i}$ are basic forms and the $t_{j}$ are birigid with $t_{i} \not \equiv t_{j} \bmod A(F)$. Then, $\varphi$ is a basic form iff $\varphi_{2}, \ldots, \varphi_{n}$ are hyperbolic, that is, 0 in $W(F)$. We call $\varphi_{1}$ the basic part of $\varphi$, if $\varphi$ is anisotropic. As a standard application of the properties of rigid elements and their behaviour under quadratic extensions (cf. [5], [6]) we get:

Proposition 2.9. Let $\varphi \cong \varphi_{1} \perp \varphi_{2} t_{2} \perp \cdots \perp \varphi_{n} t_{n}$ be an anisotropic form over $F$ where the $\varphi_{i}$ are basic forms and $t_{2}, \ldots, t_{n}$ are birigid with $t_{i} \not \equiv t_{j} \bmod A(F)$ for $i \neq j$. If $t$ is a birigid element in $F$, then $\left(\varphi_{F(\sqrt{t})}\right)_{a}$ is not universal. If $t=b t_{2}$ with $b \in A(F)$, then $\varphi_{1} \perp b \varphi_{2}$ equals the basic part of $\varphi_{a}$ in $W(F(\sqrt{t}))$.

Proposition 2.10. Let $\varphi, \psi$ be basic forms over $F$, let $t \in$ $F^{*} \backslash A(F)$, and $K:=F(\sqrt{t})$.

(i) If $\varphi \cong \psi$ over $K$, then $\varphi \cong \psi$ over $F$.

(ii) If $\varphi$ is round over $K$, then $\varphi$ is hyperbolic or anisotropic and round over $F$.

Proof. (i) $\varphi \perp-\psi$ is a basic form over $F$. From $\varphi-\psi=0$ in $W(K)$ we get $(\varphi-\psi)_{a} \cong_{F}\langle 1,-t\rangle \otimes \rho$ for an $F$-form $\rho$ (possibly $=0)$. From this we get easily $\varphi \cong \psi$ over $F$.

(ii) According to (2.9), we may assume that $\varphi_{K}$ is either hyperbolic or anisotropic. If $\varphi_{K}$ is hyperbolic, then $\varphi \cong m \times\langle 1,-1\rangle$ over $K$ and hence also over $F$, by (i). If $\varphi_{K}$ is anisotropic, then so is $\varphi_{F}$. We have $\varphi \cong x \varphi$ for $x \in D_{F}(\varphi)(\subset A(F))$ over $K$; hence by (i) $\varphi \cong x \varphi$ over $F$, i.e., $\varphi$ is round over $F$.

We now settle the non-formally real case where besides squares only birigid elements are represented. According to (2.4), we can restrict ourselves to anisotropic forms. 
LEMMA 2.11. Let $F$ be non-formally real, and let $\varphi$ be an anisotropic form over $F$ with $1 \in D(\varphi)$. Moreover, let $\varphi^{\prime} \cong \gamma \otimes\langle t\rangle$ where $\gamma$ is a basic form and $t \notin A(F)$. If $\varphi$ is round over $F(\sqrt{a})$ whenever $a \in D_{F}(\varphi)$, then $\varphi$ is round over $F$.

Proof. We may assume that $\operatorname{dim} \gamma \geq 2$ and $1 \in D_{F}(\gamma)$. Since $F$ is non-formally real, we have $\operatorname{dim} \varphi \in 2 \mathbb{N}$ (cf. [14]) and hence $\operatorname{dim} \gamma \geq$ 3 . Let $d:=\operatorname{det} \gamma$. For $a \in D_{F}(\gamma)$ we obtain $\varphi \cong\langle 1\rangle \perp a \gamma$ over $F(\sqrt{a t})$. By (2.10), $\langle 1\rangle \perp a \gamma$ is round and anisotropic over $F$ for every $a \in D_{F}(\gamma)$ since $\gamma$ is anisotropic. First assume $\operatorname{dim} \gamma \in 1+4 \mathbb{N}$. By [14, 1.1], we have $D_{F}(\langle 1\rangle \perp a \gamma) \subset D_{F}(\langle 1, a d\rangle) \forall a \in D_{F}(\gamma)$. Pick $a \in D_{F}(\gamma)$. Since $a \in D(a \gamma) \cap D(\gamma)$, we get $a \in D(\langle 1, d\rangle) \cap$ $D(\langle 1, a d\rangle) \subset D_{F}(\langle 1,-a\rangle)$. Thus, $a \in D(\langle 1,1\rangle)$, and hence $D(\gamma) \subset$ $D(\langle 1,1\rangle)$, which gives $D(\langle 1\rangle \perp \gamma) \subset D(\langle 1,1,1\rangle)$. Since $\langle 1\rangle \perp \gamma$ is round over $F$, it follows from $\langle 1\rangle \perp \gamma \cong\langle 1,1\rangle \perp \gamma^{\prime}$ and $D\left(\gamma^{\prime}\right) \subset$ $D(\langle 1,1\rangle)$ that $1 \in D\left(\gamma^{\prime}\right)$. So, $\langle 1,1,1\rangle$ is a subform of $\langle 1\rangle \perp \gamma$. But then, by Kneser's lemma, $\langle 1\rangle \perp \gamma$ is isotropic, a contradiction. Hence, $\operatorname{dim} \gamma=1$, and we are done. Now, assume $\operatorname{dim} \gamma \in 3+4 \mathbb{N}$. By $[14,1.1]$, we have $a d \in D_{F}(\langle 1,1\rangle) \forall a \in D_{F}(\gamma)$; in particular $d \in$ $D_{F}(\langle 1,1\rangle)$ since $1 \in D_{F}(\gamma)$. Thus, again $a \in D_{F}(\langle 1,1\rangle) \forall a \in D_{F}(\gamma)$ and we get a contradiction as above. This establishes the proof.

We need a simple lemma on the structure of round forms over fields with birigid elements proved in [2]:

LEMMA 2.12 (cf. $[2,3.3])$. Let $\varphi$ be a round and anisotropic form over $F$. Then, $\varphi \cong \varphi_{1} \otimes \varphi_{2}$ where $\varphi_{1}$ is a basic round form over $F$ and $\varphi_{2}$ is a Pfister form and where $\varphi_{2}^{\prime}$ represents only birigid elements.

LEMMA 2.13. Let $F$ be non-formally real and let $\varphi$ be an anisotropic form over $F$ with $1 \in D_{F}(\varphi)$ and $D\left(\varphi^{\prime}\right) \subset F^{*} \backslash A(F)$. If $\varphi$ is round over $F(\sqrt{a})$ whenever $a \in D_{F}(\varphi)$, then $\varphi$ is round over $F$.

Proof. According to (2.11), we may assume that there are birigid elements $x, y \in D_{F}(\varphi)$ with $x \not \equiv y \bmod A(F)$. Wlog let $\operatorname{dim} \varphi \geq 3$. Then, $\varphi \cong\langle 1\rangle \perp \alpha \otimes\langle x\rangle \perp \beta$, where $\alpha$ is a basic form and $D_{F}(\beta) \cap$ $x A(F)=\varnothing$. By (2.9), $\langle 1\rangle \perp \alpha \otimes\langle x\rangle$ equals the basic part of $\varphi_{a}$ over $F(\sqrt{a x})$ where $a \in D_{F}(\alpha)$. Hence, $\langle 1\rangle \perp \alpha \otimes\langle x\rangle$ is round over $F(\sqrt{a x})$ whenever $a \in D_{F}(\alpha)$, by (2.12) in case $\varphi$ is anisotropic over $F(\sqrt{a x})$ or by (2.9) otherwise. From (2.11) we get $\alpha \cong\langle 1\rangle$. Hence, $\varphi \cong\left\langle 1, x_{1}, \ldots, x_{n}\right\rangle$ where $x_{i} \notin A(F)$ and $x_{i} \not \equiv x_{j} \bmod A(F)$ for 
$i \neq j$. It suffices to prove $x_{1} x_{2} \in D_{F}(\varphi)$. According to (2.2), (2.3), we may assume $1 \not \equiv-1 \bmod F^{* 2}$. By $(2.9), \varphi_{F\left(\sqrt{x_{1}}\right)} \cong\left\langle 1,1, x_{2}, \ldots, x_{n}\right\rangle$ is anisotropic and, by $[5,2.3], x_{2}, \ldots, x_{n}$ are birigid over $F\left(\sqrt{x_{1}}\right)$. By (2.12) and [6, Prop. 5], there exists an $i \in\{3, \ldots, n\}$ such that $x_{2} \equiv x_{i} \bmod A(K)$. Hence, $x_{2} x_{i} \in A(K) \cap F=\left\{1, x_{1}\right\} A(F)$ (by [7, 5.6]). Thus, there is an $a \in A(F)$ such that $x_{i}=a x_{1} x_{2}$. Again by (2.12), we have $\langle 1,1\rangle \cong\langle 1, a\rangle$ over $F\left(\sqrt{x_{1}}\right)$, and hence $a \in F^{2}$. This completes the proof.

In the remaining "mixed" case we were not able to prove a general result. We restrict ourselves to fields whose quadratic form schemes are elementary constructible starting with schemes of non-formally real fields with $u$-invariant $\leq 2$ and the $p$-adic fields (that means the schemes can be constructed from those of the "starting fields" building product schemes and power schemes; cf. Kula [11], [12]). We use the notation of [11]. The above class of fields will be denoted by $\Omega$. For a form $\varphi$ over a product scheme $S\left(K_{1}\right) \sqcap S\left(K_{2}\right) \quad\left(K_{1}, K_{2}\right.$ fields) we write $\left(\begin{array}{l}a_{1} \cdots a_{n} \\ b_{1} \cdots b_{n}\end{array}\right)$ instead of $\left\langle\left(a_{1}, b_{1}\right), \ldots,\left(a_{n}, b_{n}\right)\right\rangle$ where $\left\langle a_{1}, \ldots, a_{n}\right\rangle$ and $\left\langle b_{1}, \ldots, b_{n}\right\rangle$ are forms over $S\left(K_{1}\right)$ and $S\left(K_{2}\right)$ respectively. We call $S\left(K_{1}\right) \sqcap S\left(K_{2}\right)$ a non-trivial product if neither $S\left(K_{1}\right)$ nor $S\left(K_{2}\right)$ is equivalent to $S(\mathbb{C})$. If $S(K) \cong S\left(K_{1}\right) \sqcap S\left(K_{2}\right)$, we identify square classes of $K$ with scheme elements of $S(K)$, and forms over $K$ with forms over $S(K)$ (cf. [2]). A power scheme of a scheme $S$ is denoted by $S^{T}$ where $T$ is an elementary abelian 2-group (for the exact definition see [11]).

Proposition 2.14. Let $F$ be a non-formally real field with $S(F) \cong$ $\left(S\left(K_{1}\right) \sqcap S\left(K_{2}\right)\right)^{T}$ where $S\left(K_{1}\right) \sqcap S\left(K_{2}\right)$ is a non-trivial product. Let $\varphi$ and $\psi$ be basic forms over $F$ with $\operatorname{dim} \varphi \geq 2$ and $1 \in D(\varphi)$. If $\varphi \cong \psi$ holds over $K=F(\sqrt{a})$ whenever $a \in D_{F}(\varphi)$, then $\varphi \cong \psi$ over $F$.

Proof. After identification we may assume

$$
\varphi \cong\left(\begin{array}{cc}
1 & a_{1} \cdots a_{n} \\
1 & b_{1} \cdots b_{n}
\end{array}\right) \text { and } \psi \cong\left(\begin{array}{c}
c_{1} \cdots c_{m} \\
d_{1} \cdots d_{m}
\end{array}\right)
$$

Since $F$ is nonreal, both $K_{1}$ and $K_{2}$ must be nonreal. According to Kneser's lemma, there are $a \in \widetilde{D}_{K_{1}}\left(\left\langle 1, a_{1}, \ldots, a_{n}\right\rangle\right) \backslash\{1\}$ and $b \in$ $\widetilde{D}_{K_{2}}\left(\left\langle 1, b_{1}, \ldots, b_{n}\right\rangle\right) \backslash\{1\}$. Hence, $\tilde{a}:=\left(\begin{array}{l}a \\ 1\end{array}\right) \in \widetilde{D}_{K}(\varphi) \backslash\{1\}$. We have 
$\varphi \cong \psi$ over $F(\sqrt{\tilde{a}})$ and over $F(\sqrt{\tilde{b}})$ as well, and hence

$$
(\varphi-\psi)_{a} \cong\left(\begin{array}{ll}
1 & -a \\
1 & -1
\end{array}\right) \otimes \rho \text { and }(\varphi-\psi)_{a} \cong\left(\begin{array}{ll}
1 & -1 \\
1 & -b
\end{array}\right) \otimes \delta
$$

over $F$ for some $F$-forms $\rho, \delta$. Since $\varphi-\psi$ is basic, the forms $\rho$ and $\delta$ are also basic, that is, they are defined over $S\left(K_{1}\right) \sqcap S\left(K_{2}\right)$. Thus,

$$
\begin{aligned}
& \varphi \perp-\psi=\left(\begin{array}{ccc} 
& * & \\
1 & -1 \ldots 1 & -1
\end{array}\right) \text { and } \\
& \varphi \perp-\psi=\left(\begin{array}{ccc}
1 & -1 \ldots 1 & -1 \\
* &
\end{array}\right)
\end{aligned}
$$

and hence $\varphi-\psi=0$.

THeOReM 2.15. Let $F \in \Omega$. If $\varphi$ is a form over $F$ with $1 \in D_{F}(\varphi)$ such that $\varphi$ is round over $F(\sqrt{a})$ whenever $a \in D_{F}(\varphi)$, then $\varphi$ is round over $F$.

Proof. According to (2.4) and (2.13), we may assume that $\varphi$ is anisotropic and of the type $\varphi \cong \varphi_{1} \perp \varphi_{2} t_{2} \perp \cdots \perp \varphi_{n} t_{n}$ where the $\varphi_{i}$ are basic forms with $1 \in D_{F}\left(\varphi_{i}\right)$ for $i=1, \ldots, n, \operatorname{dim} \varphi_{1} \geq 2$, and the $t_{i}$ are birigid with $t_{i} \not \equiv t_{j} \bmod A(F)$ for $i \neq j$. By (2.7), $\varphi_{1}$ is round over $F$ and $D\left(\varphi_{1}\right) \subset G\left(\varphi_{i}\right) \subset D\left(\varphi_{i}\right)$ for $i=2, \ldots, n$. Let $a \in$ $D_{F}\left(\varphi_{1}\right) \backslash F^{2}$ and $K=F(\sqrt{a})$. If $\left(\varphi_{1}\right)_{K}$ is isotropic, then $\varphi_{1}$ contains a subform $\langle z,-a z\rangle$ over $F\left(z \in F^{*}\right)$ and hence $-a \in D_{F}\left(\varphi_{1}\right)$. But then $-1 \in D_{F}\left(\varphi_{1}\right) \subset G_{F}(\varphi)$ and, by (2.2) and (2.3), we are done. Therefore, we may assume that $\varphi_{1}$ is anisotropic over $K=F(\sqrt{a})$ whenever $a \in D_{F}\left(\varphi_{1}\right)$. Let $j \in\{2, \ldots, n\}$. If $\left(\varphi_{j}\right)_{K}$ is isotropic, then $\langle w,-w a\rangle$ is a subform of $\varphi_{j}$ over $F$ for some $w \in D_{F}\left(\varphi_{j}\right)$ and we get $w t_{j} \in G_{F}(\varphi)$, by (2.1). This implies $\varphi_{1} \cong \varphi_{j}$ over $F$. If $\left(\varphi_{j}\right)_{L}$ is anisotropic for all $L=F(\sqrt{b})$ where $b \in D_{F}\left(\varphi_{1}\right)$, then also $\varphi_{1} \cong \varphi_{j}$ over $F$, by (2.12) and (2.14) or trivially (in case $S(F)$ is equivalent to a power scheme of a scheme of a "starting field"). Thus, we have $\varphi_{1} \cong \varphi_{2} \cong \ldots \cong \varphi_{n}$ over $F$. Now it suffices to prove $t_{i} t_{j} \in D_{F}(\varphi)$ for $i \neq j$. Wlog let $i=2, j=3$. Moreover, let $K:=F\left(\sqrt{t_{2}}\right)$. Then, $\varphi_{K}$ is round and anisotropic or hyperbolic, by (2.9). If $\varphi_{K}$ is anisotropic, we have $t_{3} \cong t_{j} \bmod A(K)$ for a $j \geq 4$, by (2.12). As in the proof of (2.13), we now get $t_{j}=a t_{2} t_{3}$ for an $a \in A(F)$. We have $\varphi_{1} \perp \varphi_{1} \cong \varphi_{1} \perp a \varphi_{1}$ over $K$, according to (2.12). Hence, $\varphi_{1} \cong a \varphi_{1}$ over $K$ and, by (2.10), $\varphi_{1} \cong a \varphi_{1}$ over $F$, so we are done. In the hyperbolic case we get $\varphi_{1} \cong-\varphi_{1}$ over $K$, by (2.9), 
and hence over $F$, by (2.10). This means $-1 \in D_{F}\left(\varphi_{1}\right) \subset G_{F}(\varphi)$, and again we are done.

We now want to examine the formally real pythagorean case.

LEMMA 2.16. Let $F$ be f.r. pythagorean and let $\varphi$ be an anisotropic form over $F$ with $1, x, y, x y \in D_{F}(\varphi)$ for some $x, y \in F \backslash F^{2}$ with $x \notin y F^{2}$. If $\varphi$ is round over $F(\sqrt{a})$ whenever $a \in D_{F}(\varphi)$, then $\varphi$ is round over $F$.

Proof. Let $P$ be an ordering of $F$. Then, at least one element of the set $\{x, y, x y\}$ is in $P$, say $x$. Now, $P$ extends to an ordering $\widetilde{P}$ over $F(\sqrt{x})$ (cf. $[16,3.1 .11])$. Since $\varphi$ is round over $F(\sqrt{x})$ we have $\operatorname{sgn}_{\widetilde{P}} \varphi \in\{0, \operatorname{dim} \varphi\}$, by $[14,1.1]$. Hence, $\operatorname{sgn}_{P} \varphi \in\{0, \operatorname{dim} \varphi\}$ for all orderings $P$ of $F$. According to the proof of [3, Satz 16], $\varphi \cong l \times \psi$ for a Pfister form $\psi$, that is, $\varphi$ is round over $F$.

COROLlaRY 2.17. Let $F$ be a f.r. pythagorean field and let $\varphi$ be an anisotropic form over $F$ with $1 \in D_{F}(\varphi)$ and $\left|\widetilde{D}_{F}(\varphi)\right|>2$. If $\varphi$ is round over $F(\sqrt{a})$ whenever $a \in\left\langle D_{F}(\varphi)\right\rangle$, then $\varphi$ is round over $F$.

CoROLlaRY 2.18. Let $F$ be a f.r. pythagorean field and let $\varphi$ be an anisotropic form over $F$ with $1 \in D_{F}(\varphi)$ such that $\varphi$ represents at least one non-rigid element. If $\varphi$ is round over $F(\sqrt{a})$ whenever $a \in D_{F}(\varphi)$, then $\varphi$ is round over $F$.

LEMMA 2.19. Let $F$ be a SAP-pythagorean field, and let $\varphi$ be an anisotropic form over $F$ with $1 \in D_{F}(\varphi)$ and $\left|\widetilde{D}_{F}(\varphi)\right|>2$. If $\varphi$ is round over $F(\sqrt{a})$ whenever $a \in D_{F}(\varphi)$, then $\varphi$ is round over $F$.

Proof. Wlog we may assume $\operatorname{dim} \varphi \geq 3$ and $\varphi \cong\langle 1, a, b, \ldots\rangle$ where $a, b \notin F^{2}$ and $a \notin b F^{2}$. By [17, Prop. 7] (or [15, Th. 3.1]), $\langle 1, a, b,-a b\rangle$ is isotropic, hence $a b \in D_{F}(\langle 1, a, b\rangle) \subset D_{F}(\varphi)$. Now, (2.16) yields the claim.

We now consider the case where at least one birigid element is represented by $\varphi$.

Proposition 2.20. Let $F$ be a f.r. pythagorean field and let $\varphi \cong$ $\alpha \perp \beta t$ be an anisotropic form over $F$ where $\alpha$ and $\beta$ are basic, $1 \in D(\alpha), D(\beta)$, and $t \in F^{*} \backslash A(F)$. If $\left|\widetilde{D}_{F}(\varphi)\right|>2$ and $\varphi$ is round over $F(\sqrt{c})$ whenever $c \in D_{F}(\varphi)$, then $\varphi$ is round over $F$. 
Proof. First assume $\left|\widetilde{D}_{F}(\beta)\right|>1$. Then, there exists a non-square $a \in D_{F}(\beta)$. Now, $\alpha \perp \beta$ is round over $F(\sqrt{t})$ and $\alpha \perp \alpha \beta$ is round over $F(\sqrt{a t})$. By $(2.10), \alpha \perp \beta$ and $\alpha \perp a \beta$ are round over $F$. Hence, $a \alpha \perp a \beta \cong \alpha \perp \beta$ and $\alpha \perp \alpha \beta \cong a \alpha \perp \beta$. From this we get $a \alpha \cong \alpha$, since $F$ is pythagorean. So $a, t, a t \in D_{F}(\varphi)$, and by (2.16) we are done. Now, assume $\widetilde{D}(\beta)=\{1\}$ and $b \in D_{F}(\alpha) \backslash F^{2}$. By (2.18), we may assume that $b$ is rigid; hence $K:=F(\sqrt{b})$ is pythagorean, by $[13,5.15]$. If $\varphi$ is isotropic over $K$, then it is hyperbolic. But this means $\beta_{K}=0$ since $\alpha, \beta$ are basic and $t$ is birigid over $K$, a contradiction. Hence, $\varphi_{K}$ is anisotropic. By (2.12), we have $\alpha_{F} \cong\langle 1, \ldots, 1, b, \ldots, b\rangle$ and $\operatorname{dim} \alpha=\operatorname{dim} \beta$. But then $\alpha \perp \beta t$ is not round over the pythagorean field $F(\sqrt{t})$. This completes the proof.

LEMMA 2.21. Let $F$ be a f.r. pythagorean field and let $\varphi$ be an anisotropic form over $F$ which represents 1 and at least one birigid element. If $\left|\widetilde{D}_{F}(\varphi)\right|>2$ and $\varphi$ is round over $F(\sqrt{a})$ whenever $a \in$ $D_{F}(\varphi)$, then $\varphi$ is round over $F$.

Proof. According to (2.20), we may assume that $\varphi$ represents birigid elements $t_{1}, t_{2}$ with $t_{1} \not \equiv t_{2} \bmod A(F)$. Let $\varphi \cong \varphi_{1} \perp \varphi_{2} t_{2} \perp \cdots \perp$ $\varphi_{n} t_{n}$ where $t_{4}=t_{2} t_{3}$, the $\varphi_{i}$ are basic forms, $t_{i} \not \equiv t_{j} \bmod A(F)$, and possibly $\varphi_{4}=0$. We may assume that $\varphi$ is anisotropic over $F\left(\sqrt{t_{2}}\right)$ and $F\left(\sqrt{t_{3}}\right)$, by (2.10). According to (2.12), we get $\varphi_{1} \perp \varphi_{2} \cong \varphi_{3} \perp$ $\varphi_{4}$ over $F\left(\sqrt{t_{2}}\right)$ and hence over $F$, by (2.10). Analogously, we get $\varphi_{1} \perp \varphi_{3} \cong \varphi_{2} \perp \varphi_{4}$ over $F$. Thus, $\varphi_{2} \cong \cong_{F} \varphi_{3}$ since $F$ is pythagorean. By cancelling, we get $\varphi_{1} \cong \varphi_{4}$, and by (2.16), we are done.

If we consider formally real fields with $u$-invariant 2 we easily get the desired result. In this case, there exists a torsion Pfister form $\langle 1, x\rangle \neq 0$, so $D(\langle 1, x\rangle)=F^{*}$. Now, (2.1) yields the desired result and we do not need the additional assumption that the form under consideration must represent more than two elements modulo $F^{* 2}$. Even if we require this, there are counter-examples in case of $u(F) \geq 4$ :

COUNTER-EXAMPLES 2.22. In the sequel we need a slight generalization of (1.3). It is immediate from [6, Th. 1, Prop. 1] that the assertion of (1.3) holds also for different linked basic Pfister forms $\varphi \neq 0, \rho$ where $D(\rho)=A(F)$. 
(1) Let $F$ be a field with $S(F) \cong\left(S(\mathbb{R}) \sqcap S\left(F_{3}\right)\right)^{T}$ where $T=\{1, t\}$. Consider the form

$$
\varphi \cong\left(\begin{array}{l}
1 \\
1
\end{array}\right) \perp\left(\begin{array}{ccc}
1 & 1 & 1 \\
1 & 1 & -1
\end{array}\right) t
$$

over $F$ (so, $\left.D_{F}(\varphi)=\left\{\left(\begin{array}{l}1 \\ 1\end{array}\right),\left(\begin{array}{l}1 \\ 1\end{array}\right) t,\left(\begin{array}{c}1 \\ -1\end{array}\right) t\right\}\right)$. Using [5, 2.3], [6, Prop. 2] and the generalized version of (1.3) it is easy to check that $\varphi$ is round over $F(\sqrt{a})$ whenever $a \in D_{F}(\varphi)$. But, according to (2.12), $\varphi$ is not round over $F$. This also shows that Proposition (2.20) does not hold in general when $u(F) \geq 4$. Moreover, since $F$ is a SAP-field, the assertion of Lemma (2.19) is not true for every SAP-field.

(2) Let $L:=\mathbb{Q}_{2}(\sqrt{-1})$, and let $\langle 1, a, b, a b\rangle$ be the unique anisotropic 4-dimensional form over $L$ (cf. [16, p. 217]). Then, $D_{L}(\langle a, b, a b\rangle)=L^{*} \backslash L^{* 2}$ and, by $[16,4.1 .7]$, for any $v \in L^{*} \backslash L^{* 2}$ there exists a $w \in L^{*}$ such that $\langle 1, a, b, a b\rangle \cong\langle 1, v, w, v w\rangle$. Now, let $F$ be a field with $S(F) \cong(S(\mathbb{R}) \sqcap S(L))^{T}$ where $T=\{1, t\}$. Define

$$
\varphi:=\left(\begin{array}{cccc}
1 & 1 & 1 & 1 \\
1 & a & b & a b
\end{array}\right) \quad \text { and } \quad \psi:=\left(\begin{array}{cccc}
1 & 1 & 1 & 1 \\
1 & -1 & 1 & -1
\end{array}\right) .
$$

Let $\left(\begin{array}{l}1 \\ z\end{array}\right)$ be any element of $\widetilde{D}_{F}(\varphi) \backslash\{1\}$. Then,

$$
\varphi \perp-\psi \cong\left(\begin{array}{ll}
1 & -1 \\
1 & -z
\end{array}\right) \otimes\left(\begin{array}{llll}
1 & 1 & 1 & 1 \\
1 & x & 1 & 1
\end{array}\right)
$$

for an $x \in L^{*} / L^{* 2}$. Hence, $\varphi \cong \psi$ over $F(\sqrt{x})$ whenever $x \in$ $D_{F}(\varphi) \backslash F^{2}$. Now, consider the form $\gamma:=\varphi \perp \psi t$ over $F$. Using the same arguments as in (1) one can check that $\gamma$ is round over any quadratic extension $F(\sqrt{z})$ where $z \in D_{F}(\gamma)$, however, by (2.12), it is not round over $F$. Note also that the form $\gamma$ represents a group. Hence, even if we use the stronger condition (2) (of the list at the beginning of this section) instead of (1), we have counter-examples.

Finally, we want to examine how the going-down property can be generalized. The appropriate generalization is to require "roundness" over $\varphi-2^{n}$-extensions (cf. (1.9)(2)). The usual induction proofs yield:

LEMMA 2.23. Let $\varphi$ be an nr-representable (resp. anisotropic) form over $F$ and let $1 \in D_{F}(\varphi)$. If $\varphi$ is round over any $\varphi$ - $2^{n}$-extension (resp. $2^{n}$-extension) of $F$ for a fixed $n \in \mathbb{N}^{*}$, then $\varphi$ is round over $F$. 


\section{REFERENCES}

[1] B. Alpers, Round quadratic forms, J. Algebra, to appear.

[2] __ Zum Transitivitätsverhalten von Kollineationsgruppen affin-metrischer und projektiv-metrischer Räume, Ph.D. Thesis, Hamburg 1988.

[3] E. Becker and E. Köpping, Reduzierte quadratische Formen und Semiordnungen reeller Körper, Abh. Math. Sem. Univ. Hamburg, 46 (1977), 143-177.

[4] L. Berman, Pythagorean fields and the Kaplansky radical, J. Algebra, 61 (1979), 497-507.

[5] __, Quadratic forms and power series fields, Pacific J. Math., 89 (1980), 257267.

[6] L. Berman, C. Cordes, and R. Ware, Quadratic forms, rigid elements, and formal power series fields, J. Algebra, 66 (1980), 123-133.

[7] R. Bos, Quadratic forms, orderings, and abstract Witt rings, Ph.D. Thesis, Utrecht, 1984.

[8] C. Cordes and J. R. Ramsey, Quadratic forms over fields with $u=q / 2<+\infty$, Fund. Math., 99 (1978), 1-10.

[9] R. Elman and T. Y. Lam, Quadratic forms and the u-invariant II, Invent. Math., 21 (1973), 125-137.

[10] __ Quadratic forms under algebraic extensions, Math. Ann., 219 (1976), 21 42.

[11] M. Kula, Fields with prescribed quadratic form schemes, Math. Z. 167 (1979), 201-212.

[12] __ Fields and quadratic form schemes, Uniw. Slaski Kat. Prace Nauk.-Prace Mat., 693 (1985) = Ann. Math. Sil., 1 (1985), 7-22.

[13] T. Y. Lam, Orderings, valuations and quadratic forms, CBMS, AMS, Providence, 1983.

[14] M. Marshall, Round quadratic forms, Math. Z., 140 (1974), 255-262.

[15] A. Rosenberg and R. Ware, Equivalent topological properties of the space of signatures of a semilocal ring, Publ. Math. Debrecen, 23 (1976), 283-288.

[16] W. Scharlau, Quadratic and Hermitian Forms, Springer, Berlin-Heidelberg-New York-Tokyo, 1985.

[17] D. Shapiro and T. Y. Lam, The square class invariant for pythagorean fields, Contemporary Math., 8 (1982), 327-340.

Received January 18, 1989 and in revised form July 6, 1989. This article was written during the author's stay as a postdoctoral fellow at the University of Saskatchewan in Saskatoon. The author would like to thank this institution, in particular Prof. Marshall, for the hospitality and the inspiring atmosphere.

UNIVERSITY OF SASKATCHEWAN

SASKATOON, SASKaTCHEWAN

S7N OW0, CANADA 



\title{
PACIFIC JOURNAL OF MATHEMATICS EDITORS
}

\author{
V. S. VARADARAJAN \\ (Managing Editor) \\ University of California \\ Los Angeles, CA 90024-1555-05 \\ Herbert Clemens \\ University of Utah \\ Salt Lake City, UT 84112 \\ THOMAS ENRIGHT \\ University of California, San Diego \\ La Jolla, CA 92093
}

R. FINN

Stanford University

Stanford, CA 94305

Hermann FlaschKa

University of Arizona

Tucson, AZ 85721

VAUGHaN F. R. JoNes

University of California

Berkeley, CA 94720

Steven Kerckhoff

Stanford University

Stanford, CA 94305

\section{C. MOORE}

University of California

Berkeley, CA 94720

Martin ScharlemanN

University of California

Santa Barbara, CA 93106

HAROLD STARK

University of California, San Diego

La Jolla, CA 92093
R. ARENS
E. F. BECKENBACH
F. WolF
K. YoshidA

\section{ASSOCIATE EDITORS}

\begin{tabular}{ll}
\multicolumn{1}{c}{ SUD-1982) } & \multicolumn{1}{c}{$(1904-1989)$} \\
UNIVERSITY OF ARIZONA & UNIVERSITY OF OREGON \\
UNIVERSITY OF BRITISH COLUMBIA & UNIVERSITY OF SOUTHERN CALIFORNIA \\
CALIFORNIA INSTITUTE OF TECHNOLOGY & STANFORD UNIVERSITY \\
UNIVERSITY OF CALIFORNIA & UNIVERSITY OF HAWAII \\
MONTANA STATE UNIVERSITY & UNIVERSITY OF TOKYO \\
UNIVERSITY OF NEVADA, RENO & UNIVERSITY OF UTAH \\
NEW MEXICO STATE UNIVERSITY & WASHINGTON STATE UNIVERSITY \\
OREGON STATE UNIVERSITY & UNIVERSITY OF WASHINGTON \\
\hline
\end{tabular}

The Supporting Institutions listed above contribute to the cost of publication of this Journal, but they are not owners or publishers and have no responsibility for its content or policies.

\begin{abstract}
Mathematical papers intended for publication in the Pacific Journal of Mathematics should be in typed form or offset-reproduced (not dittoed), double spaced with large margins. Please do not use built up fractions in the text of the manuscript. However, you may use them in the displayed equations. Underline Greek letters in red, German in green, and script in blue. The first paragraph must be capable of being used separately as a synopsis of the entire paper. In particular it should contain no bibliographic references. Please propose a heading for the odd numbered pages of less than 35 characters. Manuscripts, in triplicate, may be sent to any one of the editors. Please classify according to the 1980 Mathematics Subject Classification (1985 Revision) scheme which can be found in the December index volumes of Mathematical Reviews. Supply name and address of author to whom proofs should be sent. All other communications should be addressed to the managing editor, or Elaine Barth, University of California, Los Angeles, California 90024-1555-05.

There are page-charges associated with articles appearing in the Pacific Journal of Mathematics. These charges are expected to be paid by the author's University, Government Agency or Company. If the author or authors do not have access to such Institutional support these charges are waived. Single authors will receive 50 free reprints; joint authors will receive a total of 100 free reprints. Additional copies may be obtained at cost in multiples of 50 .
\end{abstract}

The Pacific Journal of Mathematics (ISSN 0030-8730) is published monthly except for July and August. Regular subscription rate: $\$ 190.00$ a year (10 issues). Special rate: $\$ 95.00$ a year to individual members of supporting institutions.

Subscriptions, orders for numbers issued in the last three calendar years, and changes of address should be sent to Pacific Journal of Mathematics, P.O. Box 969, Carmel Valley, CA 93924, U.S.A. Old back numbers obtainable from Kraus Periodicals Co., Route 100, Millwood, NY 10546.

The Pacific Journal of Mathematics at P.O. Box 969, Carmel Valley, CA 93924 (ISSN 0030-8730) is published monthly except for July and August. Second-class postage paid at Carmel Valley, California 93924, and additional mailing offices. Postmaster: send address changes to Pacific Journal of Mathematics, P.O. Box 969, Carmel Valley, CA 93924.

PUBLISHED BY PACIFIC JOURNAL OF MATHEMATICS, A NON-PROFIT CORPORATION Copyright (C) 1991 by Pacific Journal of Mathematics 